\title{
La alimentación en estudiantes del primer ciclo de la carrera de medicina de la Universidad Nacional de Rosario, Argentina
}

\section{Eating habits of medical students of the National University of Rosario, Argentina}

\begin{abstract}
RESUMEN
Con el objetivo de indagar sobre los hábitos alimentarios de los estudiantes de la carrera de Medicina de la Facultad de Ciencias Médicas de la Universidad Nacional de Rosario (UNR), y compararlos con las Guías Alimentarias para la Población Argentina se llevó a cabo un estudio cuali-cuantitativo, descriptivo, observacional y transversal. Se aplicó una encuesta y un recordatorio de 24 horas. La muestra quedó conformada por 140 alumnos. El consumo de alimentos declarado mostró que el 70\% había consumido leche o yogur, el 56,4\% frutas, el $79,3 \%$ verduras, el $75,7 \%$ carnes, el $62,1 \%$ cereales, el $20 \%$ huevos y el $25 \%$ fiambres. El 46,4\% declaró haber utilizado azúcar y el 20\% edulcorante. El 81,4\% expresó haber consumido panificados blancos y sólo el 15,7\% integrales. Los vegetales y el edulcorante fueron más consumidos por las mujeres y las carnes y los panificados blancos por los hombres. Respecto del hábito de desayunar fue importante el número de estudiantes que cumplió la recomendación de las Guías. El número de ingestas diarias recomendado (mínimo 4) lo cumplió el $77 \%$. Sólo el 12,8\% declaró consumir frutas y verduras tal como es recomendado (5 porciones al día). Respecto de los panificados, esta muestra no cumple la recomendación de seleccionar granos integrales.

Palabras claves: Alimentos; Estudiantes de medicina, Hábitos alimentarios, Promoción de la Salud, Recordatorio de 24 hs.
\end{abstract}

\footnotetext{
ABSTRACT

The aim of this study was to investigate the dietary habits of the students at the School of Medicine, Faculty of Medical Sciences of the National University of Rosario (UNR), and to compare them with the Dietary Guidelines for the Argentinian population. We carried out a quantitative, descriptive, observational cross-sectional study using a 24hour dietary recall questionnaire. The sample was made up of 140 students. The declared food consumption showed that $70 \%$ consumed milk or yogurt, $56.4 \%$ fruits, $79.3 \%$ vegetables, $75.7 \%$ meats, $62.1 \%$ cereals, $20 \%$ eggs and $25 \%$ cold cuts. Sugar consumption was reported by $46.4 \%$ of the students, and use of artificial sweeteners by $20 \%$ of them. Most students (81.4\%) consumed white bread and
}

Claudio Ponce', Stella M. Pezzotto 1,2 , Agustina Bertola Compagnucci ${ }^{1,2^{*}}$.

1. Área de Metodología de la Investigación Científica, Facultad de Ciencias Médicas, UNR. Rosario, Argentina.

2. Instituto de Inmunología Clínica y Experimental de Rosario. IDICER. Rosario, Argentina.

*Dirigir correspondencia a: Agustina Bertola Compagnucci. Instituto de Inmunología Clínica y Experimental de Rosario. IDICER. Suipacha 590. 2000 Rosario. Santa Fe. Argentina. Teléfono: 543414833854. E-mail: agustina_bc@hotmail.com

Este trabajo fue recibido el 17 de agosto de 2018. Aceptado con modificaciones: 15 de enero de 2019. Aceptado para ser publicado: 23 de abril de 2019 .

only $15.7 \%$ whole wheat. While vegetables and sweeteners were more consumed by women, meat and white bread were more consumed by men. Many students met the recommendation for breakfast consumption. The recommended number of daily meals (minimum 4) was met by $77 \%$. Only $12.8 \%$ reported eating fruits and vegetables as recommended (5 servings a day).Regarding breads, this sample does not meet the recommendation of selecting whole grains.

Key words: Dietary habits, Foods, Health promotion, 24 -hour dietary recall, Medical students. 


\section{INTRODUCCIÓN}

Los estilos de vida influyen de manera indudable en el estado de salud de la población, y entre sus componentes fundamentales, se encuentran los hábitos alimentarios. Es por esto que, en los tiempos actuales, la adopción de hábitos de alimentación poco saludables, fundamentalmente en el mundo occidental, han provocado graves problemas en la salud de la población, aumentando la prevalencia de enfermedades como diabetes, hipertensión arterial, obesidad, las cuales están actualmente consideradas dentro del grupo de enfermedades crónicas no transmisibles. A pesar de que los requerimientos en la alimentación se diferencian según el estado biológico en que se encuentre cada individuo entre otras tantas variables, existen actualmente guías alimentarias elaboradas en cada país, las cuales fueron llevadas a cabo teniendo en cuenta los usos y costumbres de cada región. Su finalidad es la de efectuar recomendaciones generales para la comunidad adulta sana. Dichas recomendaciones son utilizadas como una serie de propuestas básicas para fomentar en la sociedad la adopción de una conducta alimentaria saludable. Nuestro país inició a partir del año 2012, por medio de un mecanismo participativo, intersectorial y garantizando a la vez rigurosidad metodológica en su confección, un proceso de revisión de las Guías Alimentarias para la Población Argentina ${ }^{1}$, con el objetivo de obtener un mensaje consensuado y de fácil aplicabilidad.

Abordar a la alimentación en el ámbito de la universidad, constituye un paso ineludible para una educación integral, más aún en los futuros profesionales de la salud, ya que el alimentarse en forma saludable es uno de los pilares de la Promoción de la Salud. Sin embargo, cabe preguntarse si las propias prácticas alimentarias de este grupo son saludables, es decir si se aplican los conocimientos adquiridos sobre la importancia que tiene una adecuada alimentación durante los distintos períodos de la vida.

En un estudio realizado en el País Vasco en un grupo de estudiantes universitarios más del $83 \%$, no realizaba el número de comidas recomendadas ( 5 al día) además casi un $85 \%$ de los estudiantes no alcanzaba el mínimo recomendado para el grupo de las frutas y ninguno de los encuestados cumplía con las recomendaciones del consumo de verduras ${ }^{2}$. En otro trabajo llevado a cabo en el mismo país, los estudiantes de Farmacia, Enfermería y Nutrición, no realizaron una ingesta promedio adecuada de calorías y presentaban valores promedio superiores a los recomendados para el consumo de lípidos y proteínas, mientras que el consumo medio de fibras y micronutrientes se encontraba muy por debajo de lo sugerido ${ }^{3}$. En un estudio realizado en una Universidad de México, se concluyó que las dietas se caracterizan por un excesivo consumo de productos de origen animal, ricos en colesterol y grasas saturadas mientras que las cantidades incorporadas de frutas y verduras se encontraban por debajo de lo recomendado ${ }^{4}$. En un estudio realizado en Colombia se encontró que la dieta de adolescentes universitarios se caracterizó por un bajo consumo de frutas y verduras y un exceso de consumo de carbohidratos y proteínas de origen animal ${ }^{5}$. En un trabajo realizado en una universidad chilena, se encontró una alta prevalencia de sobrepeso y obesidad de los estudiantes. Como principales causas se destacaron la escasa proporción de alumnos con hábitos de alimentación saludable y la falta de actividad física ${ }^{6}$.

Existe escasa información sobre la situación nutricional y los hábitos alimentarios de los jóvenes de nuestro país, siendo este grupo poblacional uno de los más influenciables por los medios de comunicación, los cuales no tienen como prioridad el fomentar una alimentación racional y saludable. En un estudio realizado con alumnos ingresantes a la carrera de Medicina de la Universidad de Buenos Aires se reportó que aproximadamente un 38\% de los encuestados realizaban cuatro comidas diarias, pero en general en horarios irregulares ${ }^{7}$. En otro estudio realizado en la misma Universidad se incluyó a estudiantes de las carreras de Medicina y Arquitectura y se encontró que el aporte calórico fue superior al recomendado, siendo el consumo de frutas y verduras insuficiente para cubrir las recomendaciones diarias de las Guías Alimentarias para la Población Argentina ${ }^{8}$. Sin embargo, en otro estudio de origen nacional, se encontró que todos los encuestados cumplían con el consumo recomendado de lácteos y derivados ${ }^{9}$. En una presentación realizada por el Capítulo Argentino de la Sociedad Latinoamericana de Nutrición, investigadores de la Universidad Nacional de Luján informaron que las principales fuentes de carbohidratos en la alimentación de estudiantes de esa Universidad, la constituyen los tubérculos, estando el consumo de legumbres muy por debajo de lo recomendado ${ }^{10}$.

A través del presente trabajo se buscó indagar sobre los hábitos alimentarios de los estudiantes del primer ciclo de la carrera de Medicina de la Facultad de Ciencias Médicas de la Universidad Nacional de Rosario, y proceder a la comparación de dichos hábitos, con las Guías Alimentarias para la Población Argentina', las cuales fueron concebidas como un instrumento de educación para la salud, adaptando los conocimientos científicos sobre requerimientos nutricionales y composición de los alimentos, en mensajes que faciliten a la población la selección y el consumo de alimentos saludables.

\section{MATERIALES Y MÉTODOS}

Se llevó a cabo un estudio cuali-cuantitativo, descriptivo, observacional de corte transversal. La muestra quedó conformada por 140 alumnos del Ciclo Promoción de la Salud de la Carrera de Medicina de la Facultad de Ciencias Médicas de la Universidad Nacional de Rosario.

La muestra se obtuvo de manera probabilística. Se entrevistaron alumnos de primero, segundo y tercer año que cursaban a la mañana o a la tarde. El cálculo del tamaño muestral se realizó con un intervalo de confianza del 95\% y un margen de error del $8,2 \%$. Se utilizó el programa Epiinfo versión 6.0.

La técnica para la recolección de datos en este trabajo 
fue una encuesta que consta, como instrumento, de un recordatorio de 24 horas. Además, en la misma se consignaron, dentro de los datos personales, la edad y el sexo. Todos los participantes firmaron un Formulario de Consentimiento Informado a fin de aceptar su participación en el estudio. No hubo alumnos que se negaran a participar. El proyecto fue aprobado por el Comité de Bioética de la Facultad de Ciencias Médicas de la UNR.

El recordatorio de 24 horas consiste en definir y cuantificar todas las comidas ingeridas del día anterior a la realización de la encuesta. La calidad de los datos recabados con este método depende de la memoria del individuo, y como en este caso se trabaja con personas jóvenes, éste no se convierte en un obstáculo que dificulte la obtención de datos fidedignos. Este es un método sencillo y rápido, pero que requiere de ciertos cuidados en la realización del mismo para minimizar los errores. El recordatorio de 24 horas es uno de los métodos de elección en los estudios epidemiológicos transversales, que tienen como objetivo describir la ingesta alimentaria de una población determinada, paso necesario en vista a la planificación de políticas de promoción de la salud y de una nutrición saludable ${ }^{11}$. Se considera que es un instrumento rápido, económico, aplicable a la mayor parte de las poblaciones y con una alta tasa de respuesta ${ }^{11}$. Además, está comprobado que las personas con mayor nivel de instrucción tienen mejor capacidad para recordar su alimentación ${ }^{11}$.

Para este recordatorio de 24 horas se realizó una prueba piloto aplicándolo en 20 alumnos, previo al inicio de la recolección de datos.

Los alimentos consumidos fueron agrupados según características afines para poder identificar en forma sencilla cuáles son los grupos alimentarios más y menos consumidos. Se identificaron además aquellos factores asociados a la alimentación que podrían influir en los hábitos alimentarios: quién es el responsable de cocinar y con qué frecuencia acude a comprar comidas preparadas.

Finalmente, la ingesta alimentaria de la muestra estudiada, fue comparada con el contenido de los Mensajes de las Guías Alimentarias para la Población Argentina para identificar las diferencias y poder establecer posibles metas de promoción de la salud dirigidos a esta población.

Los datos obtenidos se resumieron en tablas y gráficos. Para las variables cuantitativas se obtuvieron promedios y desvíos estándar y para las cualitativas se calcularon las frecuencias absolutas y relativas. Las diferencias de consumo según sexo se compararon con el Test de Chi Cuadrado utilizando programas del paquete estadístico STATA ${ }^{12}$.

\section{RESULTADOS}

Se consignaron 140 recordatorios de 24 horas de alumnos del primer ciclo de la Carrera de Medicina de la Facultad de Ciencias Médicas de la Universidad Nacional de Rosario. De los mismos, 48 (34,3\%) correspondieron a hombres y $92(65,7 \%)$ a mujeres.

En relación a los hábitos alimentarios, fue muy alto el número de participantes que realizó el desayuno (90\%). El $55 \%$ de la muestra efectuó 4 comidas diarias, el $22 \%$, 5 o 6 comidas y el $23 \%$ restante 3 o menos ingestas a lo largo del día.

$\mathrm{Al}$ interrogar sobre la elaboración de las comidas, un $80 \%$ manifestó que las mismas eran preparadas en el hogar, siendo bajo el porcentaje de alumnos que declararon comer comidas compradas. Al consultar sobre quién es el encargado de la elaboración de los alimentos en el hogar, el $73 \%$ manifestó que ellos mismos, en el resto de los casos la realizaba algún familiar.

El consumo de alimentos declarado según la ingesta de las 24 horas previas, mostró que el $70 \%$ había consumido leche o yogur, el $56,4 \%$ frutas y el $79,3 \%$ verduras. Al clasificar los vegetales en crudos, cocidos y papas, el primer grupo fue consumido por el $45,7 \%$ de la muestra, el segundo por el $33,6 \%$ y la papa por el $26,4 \%$; cabe aclarar que algunos estudiantes consumieron más de una variedad a lo largo del día previo.

Se contabilizaron los números de porciones de vegetales y frutas consumidos por los estudiantes a lo largo del día. Un $12,9 \%$ no consumió ni frutas ni verduras. Un $47,8 \%$ ingirió una o dos porciones, el $17,9 \%$ tres porciones, el $8,6 \%$ cuatro porciones, el $6,4 \%$ cinco porciones, y seis o más porciones el $6,4 \%$.

La ingesta de carnes fue del $75,7 \%$; al clasificarlas en vacuna, pollo, pescado y cerdo, la más consumida fue la carne vacuna con un $46,4 \%$, seguida del pollo $32,9 \%$, el pescado el $9,3 \%$ y el cerdo con menos del $1 \%$. Tal como sucedió con los vegetales, debe aclararse que algunos alumnos consumieron más de una variedad de carnes en el transcurso del día.

Los cereales, pastas y granos fueron consumidos por el $62,1 \%$ de la muestra estudiada, los huevos por el $20 \%$ y los fiambres y embutidos por el $25 \%$.

El 37\% declaró haber consumido algún tipo de golosina. Para endulzar, el 46,4 \% declaró utilizar azúcar, y el 20\% edulcorante. Los panificados blancos fueron consumidos por el $81,4 \%$ y sólo el $15,7 \%$ declaró ingerir panificados integrales o con salvado.

Las diferencias de consumos de los diferentes grupos de alimentos por sexo, se observan en la tabla 1 . Se obtuvieron resultados significativos para los vegetales, que fueron más consumidos por las mujeres $(p=0,026)$ y en la clasificación de los mismos, los cocidos también fueron más ingeridos por este grupo $(p=0,021)$. Las carnes en general $(p=0,05)$ y la vacuna, luego de clasificarlas, fueron más ingeridas por los hombres $(p=0,002)$. El edulcorante fue más utilizado por las mujeres $(p=0,041)$. Y los panificados blancos más consumidos por los hombres $(p=0,007)$.

Al realizar el análisis cualitativo del perfil de consumo y los hábitos alimentarios de la muestra comparados con las recomendaciones de las Guías Alimentarias para la Población Argentina', nos encontramos con los siguientes resultados. En relación a los hábitos alimentarios fue muy importante el número de estudiantes de la muestra estudiada 
Tabla 1. Diferencias entre hombres y mujeres en el consumo de los alimentos considerados.

\begin{tabular}{|c|c|c|c|c|}
\hline ALIMENTOS & CONSUMO & HOMBRES & MUJERES & $\mathbf{P}$ \\
\hline Leche o yogur & $\begin{array}{l}\mathrm{No} \\
\mathrm{Si}\end{array}$ & $\begin{array}{l}29,2 \% \\
70,8 \%\end{array}$ & $\begin{array}{l}30,4 \% \\
69,6 \%\end{array}$ & 0,876 \\
\hline Frutas & $\begin{array}{l}\mathrm{No} \\
\mathrm{Si}\end{array}$ & $\begin{array}{l}39,6 \% \\
60,4 \%\end{array}$ & $\begin{array}{l}45,7 \% \\
54,3 \%\end{array}$ & 0,492 \\
\hline Verduras & $\begin{array}{l}\mathrm{No} \\
\mathrm{Si}\end{array}$ & $\begin{array}{l}31,3 \% \\
68,8 \%\end{array}$ & $\begin{array}{l}15,2 \% \\
84,8 \%\end{array}$ & $0,026^{*}$ \\
\hline Crudas & $\begin{array}{l}\mathrm{No} \\
\mathrm{Si}\end{array}$ & $\begin{array}{l}56,3 \% \\
43,8 \%\end{array}$ & $\begin{array}{l}53,3 \% \\
46,7 \%\end{array}$ & 0,736 \\
\hline Cocidas & $\begin{array}{l}\mathrm{No} \\
\mathrm{Si}\end{array}$ & $\begin{array}{l}79,2 \% \\
20,8 \%\end{array}$ & $\begin{array}{l}59,8 \% \\
40,2 \%\end{array}$ & $0,021^{*}$ \\
\hline Рара & $\begin{array}{l}\mathrm{No} \\
\mathrm{Si}\end{array}$ & $\begin{array}{c}72,9 \% \\
27,1 \%\end{array}$ & $\begin{array}{l}73,9 \% \\
26,1 \%\end{array}$ & 0,899 \\
\hline Carnes & $\begin{array}{l}\text { No } \\
\text { Si }\end{array}$ & $\begin{array}{l}14,6 \% \\
85,4 \%\end{array}$ & $\begin{array}{l}29,3 \% \\
70,7 \%\end{array}$ & $0,050^{*}$ \\
\hline Pollo & $\begin{array}{l}\text { No } \\
\text { Si }\end{array}$ & $\begin{array}{l}64,6 \% \\
35,4 \%\end{array}$ & $\begin{array}{l}68,5 \% \\
31,5 \%\end{array}$ & 0,641 \\
\hline Pescado & $\begin{array}{l}\text { No } \\
\text { Si }\end{array}$ & $\begin{array}{c}93,8 \% \\
6,3 \%\end{array}$ & $\begin{array}{l}89,1 \% \\
10,9 \%\end{array}$ & 0,371 \\
\hline Vacuna & $\begin{array}{l}\text { No } \\
\text { Si }\end{array}$ & $\begin{array}{l}35,4 \% \\
64,6 \%\end{array}$ & $\begin{array}{l}63,0 \% \\
37,0 \%\end{array}$ & $0,002 *$ \\
\hline Cerdo & $\begin{array}{l}\text { No } \\
\text { Si }\end{array}$ & $\begin{array}{c}100,0 \% \\
0,0 \%\end{array}$ & $\begin{array}{c}98,9 \% \\
1,1 \%\end{array}$ & 0,466 \\
\hline Cereales, pastas y granos & $\begin{array}{l}\text { No } \\
\text { Si }\end{array}$ & $\begin{array}{l}41,7 \% \\
58,3 \%\end{array}$ & $\begin{array}{l}35,9 \% \\
64,1 \%\end{array}$ & 0,502 \\
\hline Huevos & $\begin{array}{l}\text { No } \\
\text { Si }\end{array}$ & $\begin{array}{l}77,1 \% \\
22,9 \%\end{array}$ & $\begin{array}{l}79,3 \% \\
20,7 \%\end{array}$ & 0,757 \\
\hline Fiambres y embutidos & $\begin{array}{l}\text { No } \\
\text { Si }\end{array}$ & $\begin{array}{l}68,8 \% \\
31,3 \%\end{array}$ & $\begin{array}{l}78,3 \% \\
21,7 \%\end{array}$ & 0,217 \\
\hline Golosinas & $\begin{array}{l}\text { No } \\
\text { Si }\end{array}$ & $\begin{array}{l}68,8 \% \\
31,3 \%\end{array}$ & $\begin{array}{l}59,8 \% \\
40,2 \%\end{array}$ & 0,297 \\
\hline Edulcorante & $\begin{array}{l}\text { No } \\
\text { Si }\end{array}$ & $\begin{array}{l}89,6 \% \\
10,4 \%\end{array}$ & $\begin{array}{l}75 \% \\
25 \%\end{array}$ & $0,041^{*}$ \\
\hline Panificados blancos & $\begin{array}{l}\mathrm{No} \\
\mathrm{Si}\end{array}$ & $\begin{array}{r}6,3 \% \\
93,8 \%\end{array}$ & $\begin{array}{l}25 \% \\
75 \%\end{array}$ & $0,007^{*}$ \\
\hline
\end{tabular}

*Diferencias estadísticamente significativas. 
que manifestaron realizar el desayuno, cumpliendo con una de las recomendaciones de la Guías. El número de ingestas diarias recomendado (mínimo de 4) lo cumplió el 77\%, el $23 \%$ restante realizó 3 o menos comidas a lo largo del día. En relación a la selección de alimentos, considerando las recomendaciones de las Guías, la ingesta de leche o yogur fue importante en esta muestra. Las frutas fueron ingeridas sólo por el 56,4\%, a pesar de que este alimento debe siempre formar parte de la alimentación diaria. Algo similar sucedió con los vegetales, el 79\% declaró haberlos ingerido. En la clasificación de los mismos, los vegetales crudos fueron los más ingeridos (en relación con los cocidos y la papa) respetándose por consiguiente la sugerencia de las Guías de seleccionar vegetales crudos por ser menores las pérdidas de vitaminas y minerales. Los vegetales cocidos fueron más ingeridos por las mujeres probablemente por las mayores dificultades en el proceso de elaboración de los mismos. Al analizar el número de porciones de vegetales y frutas consumidas a lo largo del día, se observa que fueron muchos los alumnos que no consumieron alimentos de ninguno de los 2 grupos. Además, el 74,3\% de la muestra ingirió entre 1 a 4 porciones por día. De esta manera el $87,1 \%$ no alcanzó la recomendación de ingerir al menos 5 porciones diarias de estos grupos alimentarios. Sólo el $12,8 \%$ declaró consumir frutas y verduras en más de 5 porciones a lo largo del día.

La variedad en la ingesta de carnes es otro aspecto en el que podría vislumbrarse que no se cumple con las recomendaciones de las Guías Alimentarias para la Población Argentina'. Aunque en este estudio se realizó un único recordatorio de 24 horas, no pudiendo establecerse la frecuencia semanal de consumo, las carnes vacunas fueron mucho más ingeridas que el resto, y la ingesta de pescado, declarada por el 9,3\% de la muestra estuvo representada casi en la totalidad de los casos por productos enlatados. Asimismo, el 75,7\% declaró haber consumido algún tipo de carne y un $20 \%$ huevos, observándose que el aporte de proteínas de alto valor biológico de esta muestra fue importante.

Respecto de los panificados, en general se seleccionaron los blancos y sólo el $15 \%$ declaró la ingesta de estos productos integrales. De esta manera, se observa que no se cumple con la recomendación de seleccionar variedad y granos integrales dentro de estos productos, por el importante aporte de fibra de los mismos.

\section{DISCUSIÓN}

La dieta de un individuo define en gran medida su salud, su crecimiento y desarrollo. Las tendencias alimentarias encontradas en la población estudiada, sumadas al sedentarismo y al hábito de fumar, pueden conducir al desarrollo de patologías crónicas no transmisibles de no mediar estrategias eficaces de promoción de la salud. Consideramos que en este caso existe un hecho que agrava aún más la situación antes mencionada. La población estudiada en este trabajo la constituyen futuros profesionales médicos.
Es fundamental concientizarlos sobre lo inadecuado de sus hábitos y brindar los conocimientos y las herramientas necesarias, no sólo para modificar dichos hábitos, sino para que puedan aplicarlas en su futura profesión y desarrollarse como educadores para la salud.

El objetivo de este trabajo fue conocer los hábitos alimentarios de los estudiantes del primer ciclo de la carrera de Medicina de la Facultad de Ciencias Médicas de la UNR. La principal limitación del estudio fue el instrumento utilizado, ya que un único recordatorio de 24 horas no nos permitió cuantificar las ingestas. Sin embargo, se adaptó a la necesidad de describir la ingesta alimentaria de los estudiantes. Por ende, permitió el cumplimiento del objetivo planteado.

Como fortaleza de este estudio, se destaca que es escasa la información encontrada sobre esta temática en nuestra Universidad y más específicamente en la Facultad de Ciencias Médicas, más aún si tenemos en cuenta que los alumnos al rendir todas las áreas del ciclo estudiado acceden al Título Intermedio de Promotores de Salud.

Vale la pena mencionar que los diferentes trabajos realizados en relación a este tema han considerado diferentes recomendaciones. Esto implica que las comparaciones de los resultados obtenidos con los alcanzados por otros autores deben realizarse en función de resultados absolutos.

Esta área de investigación ha sido abordada en universidades de todo el mundo. Y en particular en Latinoamérica es importante el número de trabajos hallados. Nuestros resultados en relación a la realización del desayuno fueron superiores a los encontrados en otros estudios. En Croacia, en una muestra de estudiantes universitarios, esta comida la realizaban irregularmente ${ }^{13}$. $\mathrm{Y}$ en un estudio realizado en España, en la Universidad de San Pablo, en Madrid, desayunaban el $78-90 \%$ de 3 de las 4 carreras en las que se desarrolló el estudio ${ }^{3}$.

Respecto del número de comidas realizado por la muestra, el porcentaje que efectuó 4 comidas diarias, fue superior en comparación con un estudio realizado en una Universidad en Corrientes ${ }^{7}$ y con una muestra de 274 alumnos de la Universidad Juárez Autónoma de Tabasco en México, en la cual el 50\% realizaba solo 3 comidas diarias ${ }^{4}$. Un trabajo realizado en Colombia, encontró que era muy importante el número de comidas salteadas por los estudiantes ${ }^{5}$. Valores superiores se encontraron en una gran muestra de la Universidad del País Vasco, donde el 86,6\% de la muestra consumía 5 comidas al día ${ }^{2}$. Sin embargo, en otro estudio realizado en España, en la Universidad de San Pablo, en Madrid, sólo el $12 \%$ realizaba 5 comidas diarias y el $43 \%, 3$ comidas diarias ${ }^{3}$.

Tal como obtuvimos en nuestro trabajo, se encontró un predominio de ingesta en los hogares en un estudio realizado en una universidad privada de la ciudad de Buenos Aires; el $86,7 \%$ manifestó que consumía las comidas en su hogar ${ }^{9}$. La diferencia con este trabajo, radicó en que en nuestra muestra los propios estudiantes eran quienes preparaban su comida, mientras que, en el trabajo realizado en Buenos Aires, la 
opción más frecuente era que un familiar se encargaba de la elaboración de las comidas. Esta observación es probable que se deba a que en nuestra facultad existen muchos estudiantes provenientes de otras localidades y que, por consiguiente, no viven con sus familias. Existe una tendencia de consumo respecto a que el desayuno y la cena se realice en los hogares, mientras que el almuerzo y la merienda sean realizados en la facultad, siendo éstas dos últimas las comidas que más se salteaban, tal como se destaca en el trabajo realizado en la Universidad de Pernambuco en Brasil ${ }^{14}$.

Nuestros resultados obtenidos en relación a la alta ingesta o predominio en el consumo de carnes rojas en relación a las otras carnes, fue similar a lo hallado en un estudio realizado con los estudiantes de la Universidad de Luján ${ }^{15}$, en una Universidad Privada de Buenos Aires ${ }^{9}$ y en la Universidad de Corrientes ${ }^{7}$. En la muestra de ingresantes a la carrera de medicina de la Universidad Nacional del Nordeste, también las carnes rojas fueron más consumidas que las blancas. Además, junto con las pastas fueron los alimentos más consumidos. Por el contrario, las legumbres fueron los alimentos menos consumidos junto con las carnes blancas?.

El análisis de la ingesta de frutas y verduras ha sido uno de los aspectos más tratados en todos los trabajos encontrados sobre el consumo alimentario en estudiantes universitarios. De los estudios realizados en la Argentina, en la Universidad Nacional de Luján ${ }^{15}$ se encontró que la ingesta de frutas y verduras de los estudiantes fue baja. Sin embargo, ellos encontraron un alto porcentaje de estudiantes que nunca consumían estos grupos de alimentos, en nuestra muestra solo un $12,9 \%$ declaró no consumir ni frutas ni verduras. De esta manera se observa que nuestros resultados son similares a los de la Universidad de Corrientes, donde sólo el $10,2 \%$ de los alumnos, no consumió vegetales y frutas?.

En relación al cumplimiento de las recomendaciones de consumir cuatro o más porciones diarias, en nuestra muestra lo cumplió el 21,4\%. En los estudios realizados en otros países, se observa una tendencia similar. En la Universidad de Goteborg, Suecia, sólo el 30\% de la muestra consumió más de $500 \mathrm{~g}$ de frutas y verduras, cercano a los valores obtenidos por nuestro equipo ${ }^{16}$. En la Universidad del País Vasco el $84,9 \%$ no cumplió con las recomendaciones de frutas y ninguno la de verduras ${ }^{2}$. En Colombia, solo el 17\% consumía frutas y verduras ${ }^{5}$. En la Universidad Católica de Chile, los alimentos consumidos con más frecuencia por los estudiantes eran los del grupo de comida rápida y golosinas siendo los alimentos ricos en fibra, entre ellos frutas y verduras, la última opción para la compra; a pesar de que el servicio de alimentación provisto por la Universidad cumplía con los requerimientos de horario, comodidad y variedad de alimentos saludables ${ }^{17}$. Por ende, la ingesta observada de frutas y verduras en nuestra muestra sería superior a la encontrada en otros grupos de estudiantes y con valores similares a los encontrados en los estudiantes universitarios de Suecia.
En esta muestra el consumo declarado de lácteos fue importante, similar a lo encontrado en la investigación realizada en la Universidad privada de Buenos Aires ${ }^{9}$. En este último caso fueron los lácteos enteros los más consumidos. En el trabajo realizado con los ingresantes a la carrera de medicina, el 39\% declaró nunca consumir lácteos a diferencia de los ingresantes a la carrera de medicina de la Universidad del Nordeste donde este valor fue del $8 \%{ }^{7}$.

Los cereales, pastas y granos fueron consumidos por un alto porcentaje de la muestra estudiada, y hay un predominio de consumo de los panificados blancos en relación con los integrales. Pareciera ser una tendencia propia del patrón de consumo alimentario argentino, ya que en la muestra de estudiantes de la Universidad de Luján los derivados del trigo representaron el $90 \%$ de la ingesta de cereales ${ }^{15}$. Además, al igual que lo encontrado en la muestra estudiada, en esta universidad estos alimentos también fueron más consumido por los hombres.

Los alimentos incluidos dentro del grupo de los azúcares (golosinas y el azúcar) fueron encontrados con altas ingestas en esta muestra. Vale destacar que el azúcar es generalmente más seleccionado que el edulcorante a la hora de endulzar las infusiones. En un trabajo de estudiantes de las carreras de la Facultad de Medicina de la Universidad de Buenos Aires, los autores encontraron un alto consumo de azúcares simples ${ }^{8}$.

Se encontraron muy pocos trabajos donde se analizaron las diferencias de consumo por sexo. Se encontró un único trabajo donde hallaron que la ingesta de vegetales y frutas fue mayor en las mujeres de Suecia ${ }^{16}$. Los resultados de este estudio fueron similares a los nuestros, ya que las mujeres presentaron mayores ingestas de vegetales en relación con los hombres. En la Universidad País Vasco, las mujeres presentaron mejores puntajes de la calidad de la dieta que los hombres ${ }^{2}$.

Los estudiantes de las carreras relacionadas con la salud, debieran ser los que tengan internalizados hábitos más saludables que en otras. Sin embargo, tal como han encontrado otros estudios, no se han hallado diferencias en la calidad de la alimentación entre los alumnos de las carreras afines a la salud y los de otras carreras ${ }^{5}$.

En esto, probablemente influyan dos elementos fundamentales. En primer lugar, los patrones de consumo alimentarios propios del país, que se perpetúan en las generaciones más jóvenes. Tal como vemos en nuestros resultados, el predominio de la ingesta de carnes rojas, azúcar y panificados blancos, son consumos arraigados en la sociedad, asociados a aspectos culturales propios de la Argentina. El segundo factor, que consideramos que se asocia al perfil de los estudiantes de nuestra casa de estudio es que la mayoría de ellos no vive con sus familiares, ya que debieron mudarse a la ciudad para estudiar. Esto genera que se decidan por opciones de fácil elaboración al momento de alimentarse. Se destaca que aquellas preparaciones más complejas, como en el caso de los vegetales cocidos, fueron más ingeridos por las mujeres, quienes por cuestiones 
culturales suelen aprender en sus hogares a cocinar.

Podemos concluir que la muestra estudiada cumple parcialmente con las recomendaciones de las Guías Alimentarias para la Población Argentina y que conocer esta realidad puede ser un paso inicial ineludible para la promoción de hábitos alimentarios saludables en nuestra casa de estudio.

\section{BIBLIOGRAFÍA}

1. Argentine Association of Dieticians and Nutritionist. Ministry of Health. Dietary Guidelines for the Argentine Population. Second edition. Lema S, editor, Buenos Aires, Argentina, 2016. http://www.msal.gob.ar/images/stories/ bes/graficos/0000000817cnt-2016-04_Guía_Alimentaria_ completa_web.pdf.

2. Arroyo Izaga M, Rocadio PA, Ansotegui Alday L, Pascual Apaluzza E, Salces Beti I, Rebato Ochoa E. Diet quality, overweight and obesity in university. Nutr Hosp. 2006; 21 (6): 673-679.

3. Montero-Bravo A, Ubeda Martín N, García González A. Evaluation of dietary habits of a population of university students in relation with their nutritional. Nutr Hosp. 2006;21 (4): 466-473.

4. Rivera Barragán, MdR. Hábitos alimentarios en estudiantes de la Universidad Juárez Autónoma de Tabasco. Revista Cubana de Salud Pública [Internet]. 2006;32(3). Recuperado de: http://www.redalyc.org/articulo.oa?id=21420864005

5. Tobar-Vargas LF, Vásquez-Cardoso S, Bautista-Muñoz LF. Description of food habits of faculty of Science students in the Pontificia Universidad Javeriana. Univ Sci. 2008; 13 (1): 55-64.

6. Mac Millan K. Evaluation of eating habits and physical activity patterns and nutritional status in Valparaiso Catholic University students. Rev Chil Nutr. 2007; 34(4): 330-336.

7. Gerometta H, Carrara C, Alberto Galarza L, Feyling V. Frequency of consumption of food in students entering the medical career. Rev Posgrado de la VI Cátedra de Medicina. 2004; 13: 9-13.
8. Vázquez MB, Witriw AM, Reyes Toso C. Preliminary study on dietary intake among students in the careers of medicine and architecture at the University of Buenos Aires. Diaeta. 2010; 28 (131): 14-17.

9. Sagués Casabal Y, Ammazzini GE, Ayala $M$, Cetrangolo MP, Martello ML, Sobol D, Llanos P, Frechtel G, Salinas R. Alimentary habits and risk factors in university students from the city of Buenos Aires. Actualización en Nutr. 2009; 10 (1): 49-57.

10. Marchesich C, Cabrera M, Martín MC, Medanich M. Contribution of the different food groups to the intake of carbohydrates in diets of university students. At the XXXV Annual Meeting of the Argentine Chapter of the Latin American Nutrition Society September 2012. https://docplayer.es/75584320-Xxxv-reuniondel-capitulo-argentino-de-la-sociedad-latinoamericana-denutricion-caslan.html

11. Serra Majen L, Aranceta J. Nutrition and Public Health: Methods, scientific bases and applications. Second edition.Masson Editorial, Barcelona, 2007. https://www.elsevier.com/books/ nutricion-y-salud-publica/serra-majem/978-84-458-2191-6

12. STATA 8.0 (Statistics/Data Analysis. Stata Corporation). https://www.stata.com

13. Satalic Z, Baric IC, Keser I. Diet quality in Croatian university students: energy, macronutrient and micronutrient intakes according to gender. Int J Food Sci Nutr 2007; 58(5): 398-410.

14. Martins Bion F, de Castro Chagas MH, de Santana Muniz G, Oliveira de Souza LG. Nutritional status, anthropometrical Measurements, socio-economic status, and physical activity in Brazilian University students. Nutr Hosp. 2008; 23(3): 234-241.

15. Pacin A, Martínez E, Martín de Portela ML, Neira MS. Food consumption and intake of several nutrients in a population of the University of Luján, Argentina. Arch Latinoam Nutr. 1999; 49(1): 31-39.

16. Tengvall M, Lars E. Dietary intake in Swedish medical students. Scand J Food Nutr. 2007; 51 (2): 79-84.

17. Espinoza OL, Rodríguez RF, Gálvez CJ, Mac Millan KN. Eating habits and physical activity In university students. Rev Chil Nutr. 2011. 38 (4): 458-465. 